\title{
Gap formation and soft phonon mode in the Holstein model
}

\author{
D. Meyer* and A. C. Hewson \\ Department of Mathematics, Imperial College, London, SW7 2BZ, UK \\ R. Bulla \\ Theoretische Physik III, Elektronische Korrelationen und Magnetismus, \\ Universität Augsburg, 86135 Augsburg, Germany
}

(Dated: November 13, 2018)

\begin{abstract}
We investigate electron-phonon coupling in many-electron systems using dynamical mean-field theory in combination with the numerical renormalization group. This non-perturbative method reveals significant precursor effects to the gap formation at intermediate coupling strengths. The emergence of a soft phonon mode and very strong lattice fluctuations can be understood in terms of Kondo-like physics due to the development of a double-well structure in the effective potential for the ions.
\end{abstract}

Despite the many years of study of the electron-phonon interaction in metallic systems, there remain fundamental problems that have yet to be resolved; particularly in the strong-coupling regime and in conjunction with strong electron-electron interactions. A solution to these problems will be required to understand fully phenomena such as the colossal magnetoresistance effect in manganites [1]. Also in the metallic alkaline-doped $\mathrm{C}_{60}$-based compounds, high critical superconducting temperatures have been observed 22. These materials are known to have strong electron-phonon and electron-electron interactions [2]. Recent photoemission experiments indicate strong electron-phonon coupling in the cuprate hightemperature superconductors [3]. There is a clear need of theoretical techniques to tackle these problems in the strong-coupling regime.

Although electron-phonon problems involving one or few electrons can be solved to very high accuracy 田, 5, so far there are no comparably accurate approaches for the many-electron case relevant to metallic systems. In this letter we study the simplest realization of electronphonon coupling: The Holstein model with finite electron density describes the coupling of Einstein (LO) phonons to the density of electrons of a non-degenerate conduction band:

$H=\sum_{\vec{k} \sigma} \epsilon(\vec{k}) c_{\vec{k} \sigma}^{\dagger} c_{\vec{k} \sigma}+\sum_{i} \omega_{0} b_{i}^{\dagger} b_{i}+\sum_{i} g\left(b_{i}^{\dagger}+b_{i}\right) \sum_{\sigma}\left(n_{i \sigma}-\frac{1}{2}\right)$

No general exact solution of this model is known for systems with finite electron density, even in the limit of infinite spatial dimensions $(d=\infty)$. This limit takes local quantum fluctuations fully into account and has proved to be a powerful tool in understanding strongly correlated systems [6, ,7]. Although exactly solvable for $d=\infty$, the case of a single electron in the band [5] is physically very different from the many-electron case since no electron-electron pairing (bipolaron formation, superconductivity etc.) can occur. Another, more instructive limiting case is the static limit $\omega_{0}=0$, where the phonons are replaced by a static displacement of the lattice ('static' or 'classical' approximation). Extensive calculations in this limit for $d=\infty$ have been presented in Ref. 8. However, it is immediately clear that this static limit neglects all possible effects stemming from the quantum nature of the lattice excitations. In the opposite limit of $\omega_{0} \rightarrow \infty$ the lattice reacts instantaneously to the state of the electrons. Here, the Holstein model can be mapped onto a non-retarded attractive Hubbard model [9] by integrating out the phonons. The Hubbard model has been intensively studied, and much recent progress has been based on using the $d=\infty$ limit. Although the large- $\omega_{0}$ limit is not physically relevant, it is still a useful point of reference for getting an overall understanding of the physics of the model. Of physical concern for applications are relatively small phonon frequencies of the order of $\omega_{0} \approx 0.01 W-0.2 W$ ( $W$ is the width of the electron band). In this regime, the MigdalEliashberg diagrammatic approach has been used [10]. The main feature of this approach is the neglect of vertex corrections. A sufficient criterion for its application is usually $\omega_{0} / W \ll 1$. However, at least for half-filling there is evidence that this approach breaks down for intermediate coupling strengths $g$ 10, 11]. There have also been a number of perturbative schemes going beyond the Migdal-Eliashberg approach and including some vertex $\frac{1}{2}$ ).orrections $[9,12,13,14]$.

In the dynamical mean-field theory (DMFT) a latticemodel is mapped onto an associated impurity model. The parameters of the associated impurity model are related to the Green's function of the lattice model by a selfconsistency condition. This mapping becomes exact in the limit of infinite spatial dimensions $(d=\infty)$. The method is described in detail in Ref. 7. One of the most precise techniques for solving the associated impurity model for low temperatures is the numerical renormalization group (NRG) 1 15, 16, 17. It is capable of resolving very low energy scales, and gives information about the excitation spectrum over the whole real-energy axis. The combination of DMFT and NRG has helped to solve a 


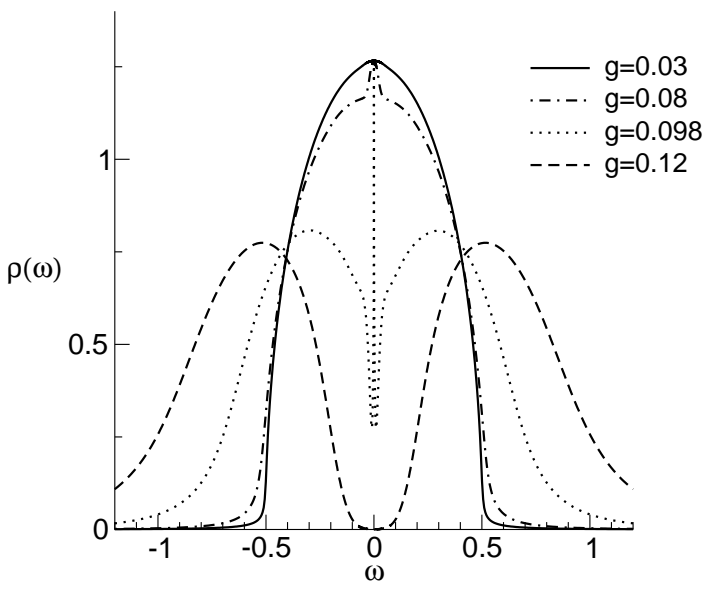

FIG. 1: Electronic spectral density $\rho(\omega)=-\frac{1}{\pi} \operatorname{Im}\left\langle\left\langle c_{i \sigma} ; c_{i \sigma}^{\dagger}\right\rangle\right\rangle$ from the DMFT-NRG calculation for $\omega_{0}=0.05$ and various coupling strengths $g$.

number of open questions regarding the Mott transition in the Hubbard model [7, 18, 19. The application of the DMFT to the Holstein model (1) leads to the AndersonHolstein impurity model, which is essentially an Anderson model with additional coupling of a local phonon mode to the impurity site:

$$
\begin{aligned}
H= & \sum_{\sigma} \epsilon_{f} f_{\sigma}^{\dagger} f_{\sigma}+g\left(b^{\dagger}+b\right) \sum_{\sigma}\left(f_{\sigma}^{\dagger} f_{\sigma}-\frac{1}{2}\right) \\
& +\sum_{\mathbf{k}, \sigma} V_{\mathbf{k}}\left(f_{\sigma}^{\dagger} c_{\mathbf{k} \sigma}+c_{\mathbf{k} \sigma}^{\dagger} f_{\sigma}\right)+\sum_{\mathbf{k} \sigma} \epsilon_{\mathbf{k}} c_{\mathbf{k} \sigma}^{\dagger} c_{\mathbf{k} \sigma}+\omega_{0} b^{\dagger} b
\end{aligned}
$$

An extensive study and discussion of this model is presented in Ref. 20, which also gives details of the generalization of the NRG to this situation 21]. In this letter, we present and discuss results obtained for the (lattice) Holstein model (1) using the NRG in conjunction with the DMFT.

We calculate the single-electron spectral function selfconsistently within the DMFT approach. The local phonon propagator $d(\omega)=\left\langle\left\langle b_{i}, b_{i}^{\dagger}\right\rangle\right\rangle$ for the Holstein model as well as spin- and charge susceptibilities for the associated impurity model can easily be obtained by this method. The phonon propagator $d(\omega)$ can also be calculated from the charge susceptibility of the impurity model:

$$
d(\omega)=d_{0}(\omega)+g^{2} d_{0}(\omega)^{2} \chi_{c}(\omega),
$$

where $d_{0}(\omega)=\left(\omega-\omega_{0}+i 0^{+}\right)^{-1}$ is the phonon propagator for $g=0$, and $\chi_{c}(\omega)$ is the charge susceptibility of the associated impurity model. Another frequently discussed phonon propagator, $D(\omega)=\left\langle\left\langle\left(b_{i}+b_{i}^{\dagger}\right),\left(b_{i}+b_{i}^{\dagger}\right)\right\rangle\right\rangle$ can be calculated by a similar formula, which is obtained by replacing $d_{0}(\omega)$ by $D_{0}(\omega)=2 \omega_{0} /\left(\omega^{2}-\omega_{0}^{2}+i 0^{+}\right)$in Eq. (3).

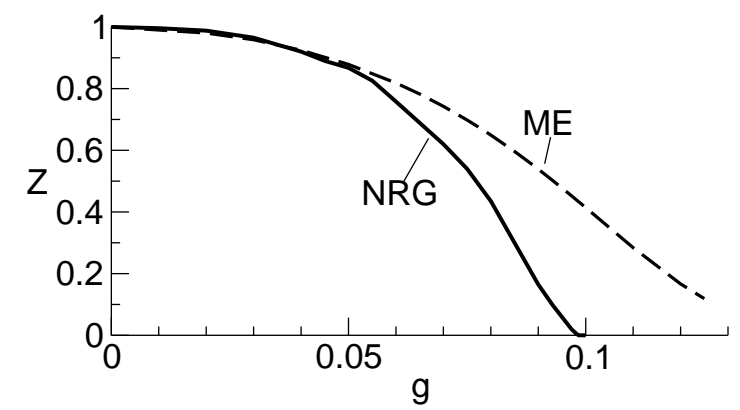

FIG. 2: Quasiparticle weight $Z=\left(1-\left.\frac{\partial \Sigma(\omega)}{\partial \omega}\right|_{\omega=0}\right)^{-1}$ as function of $g$ from the NRG and the Migdal-Eliashberg (ME) calculation

In the following numerical results, we chose for the uncorrelated system $(g=0)$ a semielliptic density of states for the conduction band. Its width $W=1$ defines the energy unit used throughout this paper. We only consider the particle-hole symmetric case and, in analogy to the Mott-transition in the Hubbard model in $d=\infty$, suppress long-range order, which corresponds to suppressing anti-ferromagnetic order in the Hubbard model. Unless otherwise noted, the phonon frequency is taken to be $\omega_{0}=0.05$. All calculations were performed for $T=0$, but our method can be extended to finite temperatures [19].

In Fig. 1, the electronic spectral function is plotted for various values of $g$ with $\omega_{0}=0.05$. For weak coupling, a small feature emerges at the Fermi energy $(\omega=0)$. With increasing $g$, this peak becomes narrower and more pronounced. This behaviour is qualitatively similar to that found within the Migdal-Eliashberg (ME) approach [10], the quantitative difference is the enhanced narrowing in the NRG calculations.

At intermediate coupling, the central feature becomes very narrow, and two broad peaks emerge above and below the Fermi energy. These are entirely absent in the ME approach. At some critical coupling $g_{c} \approx 0.099$, the central peak disappears and a gap opens between the two upper and lower bands [22]. For all $g<g_{c}$, the system is a Fermi liquid with $\operatorname{Im} \Sigma(\omega) \sim \omega^{2}$ for small energies. This manifests itself in Fig. 1 in the pinning of the spectral function at the Fermi energy.

The quasiparticle weight $Z=\left(1-\left.\frac{\partial \Sigma(\omega)}{\partial \omega}\right|_{\omega=0}\right)^{-1}$ is shown in Fig. 2 as obtained within the NRG and the ME calculation. In both cases, $Z$ decreases with increasing $g$. Up to $g \approx 0.05$ both lines coincide, but then the NRG curve decreases faster. The ME calculation breaks down before $Z$ reaches 0.1 .

We take a closer look at the large $\omega_{0}$ limit in Fig. 3, where the electronic spectral function for $\omega_{0}=1$ and 3 are plotted for three values of $g$ well below, close to, and above $g_{c}$. The two broad bands discussed above for $\omega_{0}=0.05$ split now into two each. For $\omega_{0}=3$, the 

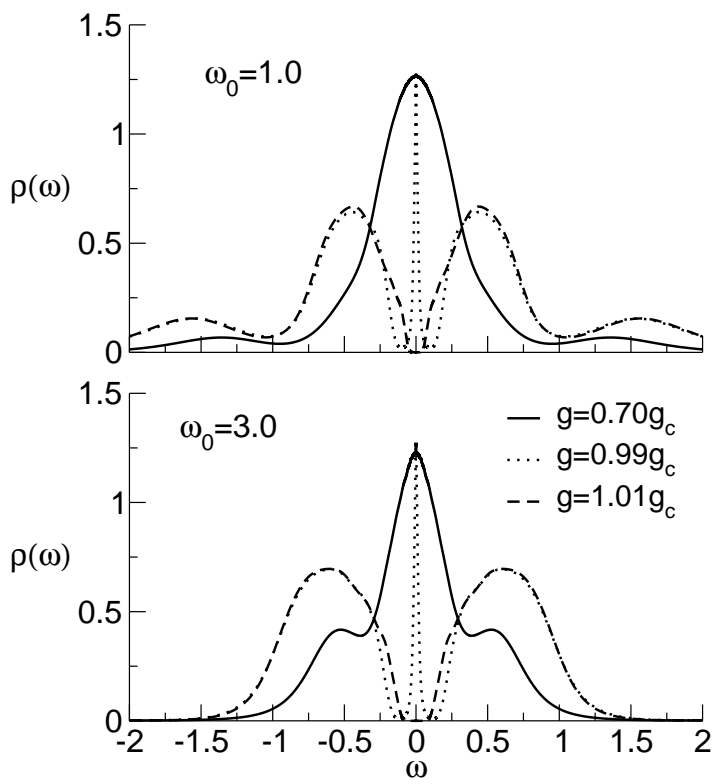

FIG. 3: Electronic spectral function as in Fig. 1, but for larger $\omega_{0}=1$ and 3 . The values of $g$ are given in units of $g_{c}$ which is $g_{c}=0.69(1.48)$ for $\omega_{0}=1(3)$.

higher-energy ones are not visible any more on the plotted scale. These bands are multiphonon bands and are shifted approximately by $\omega_{0}$. The lower-energy ones are bipolaron bands located at the bipolaron binding energy $\lambda=2 g^{2} / \omega_{0}$. The weight of the multiphonon bands vanishes with increasing $\omega_{0}$ [20], and for sufficiently large $\omega_{0}$, they can be neglected. The remaining excitation spectrum corresponds to that of an attractive Hubbard band where the effective interaction $|U|=\lambda$ corresponds to the bipolaron binding energy.

The Mott metal-insulator transition in the Hubbard model shows a parameter regime with $U_{c 1}<U<U_{c}=$ $U_{c 2}$ where metallic and insulating solutions co-exist ('hysteresis'). For large $\omega_{0}$, the effective $\left|U_{c 1,2}\right|=2 g_{c 1,2}^{2} / \omega_{0}$ of the Holstein model coincide with the values known from the Hubbard model [18]. For smaller $\omega_{0}$, the hysteresis region shrinks, and finally, for $\omega_{0}=0.05$ no hysteresis is detectable $\left(g_{c 1}=g_{c 2}\right)$.

To gain further insight into the results presented so far, let us look at the mean-field solution of model (1). The classical field $x$ is self-consistently determined as $x=\frac{1}{\sqrt{2 \omega_{0}}}\left\langle b+b^{\dagger}\right\rangle=-\sqrt{\frac{2}{\omega_{0}}} \frac{g}{\omega_{0}}\left\langle\sum_{\sigma}\left(n_{\sigma}-\frac{1}{2}\right)\right\rangle$. For small $g$ the excitation spectra remain unchanged from the $g=0$ case since any effects of the distortion are cancelled out by a change in the chemical potential. The system becomes unstable towards charge-order at a critical coupling $g_{c}^{(\mathrm{mf})}$ which for $\omega_{0}=0.05$ is $g_{c}^{(\mathrm{mf})}=0.085$. If one (artificially) restores the symmetry, one obtains an electronic excitation spectrum consisting of 2 peaks shifted by $g x_{\mathrm{mf}}$ above and below the Fermi energy. The self-consistently calcu-

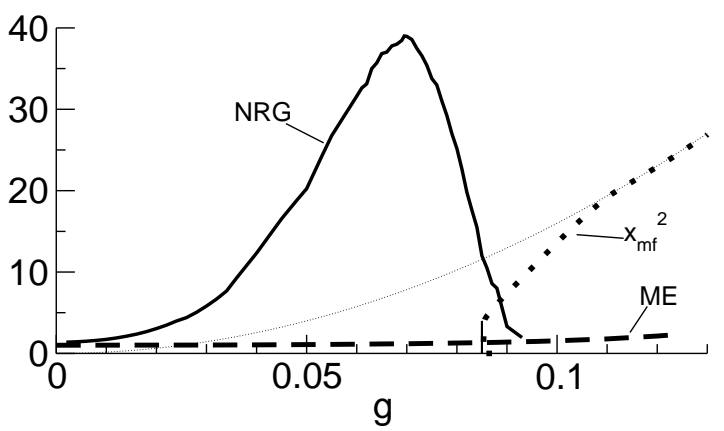

FIG. 4: Lattice fluctuations $\left\langle\hat{x}^{2}-\langle\hat{x}\rangle^{2}\right\rangle$ for NRG and MigdalEliashberg (ME) calculation. Additionally the value of $x_{\mathrm{mf}}^{2}$ as obtained within the mean-field calculation is plotted using a thick dotted line. The thin dotted line shows the limiting behaviour of $x_{\mathrm{mf}}^{2}$ for large $g, 2 \frac{g^{2}}{\omega_{0}^{3}}$.

lated values of $x_{\mathrm{mf}}^{2}$ are shown in Fig. 国 together with the asymptotic behaviour for large $g, x_{\mathrm{mf}}^{2} \rightarrow 2 g^{2} / \omega_{0}^{3}$.

This behaviour can be explained by thinking in terms of an effective potential for the ions $V(x)$. For $g<g_{c}^{(\mathrm{mf})}$, the $V(x)$ is a simple harmonic potential. For $g>g_{c}^{(\mathrm{mf})}$, it changes into a double-well structure with minima at $x_{1,2}= \pm g x_{\mathrm{mf}}$. In the mean-field approximation no fluctuations between these minima occur. To go beyond this, one needs to include these lattice fluctuations between the two minima, which has been considered in Ref. 12 .

The magnitude of the lattice fluctuations, $\left\langle\hat{x}^{2}-\langle\hat{x}\rangle^{2}\right\rangle$ is plotted in Fig. . Within the NRG calculation, this quantity has a clear maximum at a value $g^{*}\left(<g_{c}\right)$. At $g_{c}$, the fluctuations are already significantly reduced. The maximum occurs in the crossover region from a singleto the double-well potential, where the effective potential is broad and shallow. The potential barrier then grows rapidly with increasing $g$. The corresponding fluctuations in the ME calculation are always small.

Figure 5 shows the phonon propagator $\operatorname{Im} d(\omega)$ both for the Migdal-Eliashberg and the DMFT-NRG calculation. The two methods give completely different pictures. In the ME approach, the phonon propagator, which for $g=0$ consists of a peak at $\omega=\omega_{0}$, remains a single peak which softens with increasing coupling strength. In contrast to that, the NRG result shows that the main peak at $\omega=\omega_{0}$ broadens, but remains essentially unshifted. In addition, a second phonon mode appears at lower energy with increasing coupling strength. As $g$ approaches $g_{c}$ this mode softens and diverges at $g=g_{c}$. In the insulating phase, only the peak at $\omega=\omega_{0}$ remains and narrows. This behaviour in the insulating phase is to be expected as the opening of the gap inhibits any broadening due to electron-hole excitations.

Closer inspection of Figs. 4 and a shows that the soft phonon mode develops for $g \approx g^{*}$, corresponding to the maximum in $\left\langle\hat{x}^{2}-\langle\hat{x}\rangle^{2}\right\rangle$. This soft mode thus coincides 

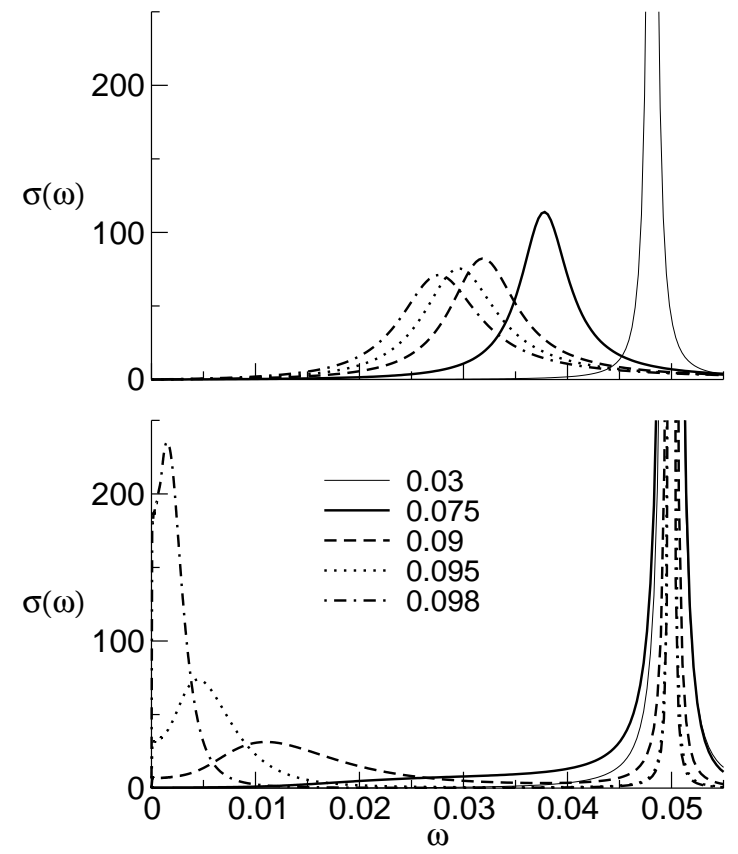

FIG. 5: Phonon propagator $\sigma(\omega)=-\frac{1}{\pi} \operatorname{Im} d(\omega)$ for $\omega_{0}=0.05$ and various coupling strengths $g$. The upper panel shows the Migdal-Eliashberg, the lower panel the NRG results.

with the build-up of the potential barrier in the effective potential of the phonons. From Eq. (3) it follows directly that the phonon propagator is closely related to the charge susceptibility of the associated impurity model. The peak in $\operatorname{Im} d(\omega)$ has its equivalence in the low-energy peak of $\chi_{c}$. The existence of such a low-energy peak can be expected as follows from the mapping of the Holstein onto an attractive Hubbard model. As noted before, the physics of the attractive Hubbard model correspond exactly to those of a repulsive Hubbard model with the spin- and charge-channels exchanged. And the latter should have a low-energy peak in the spin susceptibility due to the Kondo physics of its associated impurity model. The physics of the gap formation, and its precursor regime in the Holstein model are thus dominated by many-body physics. The low-energy feature in the phonon propagator has not been predicted before.

In this letter we have presented the application of the dynamical mean-field theory in combination with the numerical renormalization group to the Holstein model at half-filling. This method can be applied to essentially all parameter regions of the model. We studied the gap formation for small and large phonon frequencies. Generally, the gap formation has precursor effects due to many-body 'Kondo-like' physics: very strong lattice fluctuations indicate formation of a double-well potential for the ions, and a soft phonon mode emerges due to fluctuations between the two states of the system. It might be possible to observe it experimentally. The framework of our method can be extended to contain other local interactions such as electron-electron interaction of Hubbardtype to describe fullerides, or spin-exchange interactions as used to describe the manganites.

We wish to thank the EPSRC (Grant GR/J85349) and the DFG (SFB 484) for financial support, and D. M. Edwards and J. Freericks for stimulating discussions.

d.meyer@ic.ac.uk

[1] A. J. Millis, P. B. Littlewood, and B. I. Shraiman, Phys. Rev. Lett. 74, 5144 (1995).

[2] O. Gunnarson, Rev. Mod. Phys. 69, 575 (1997).

[3] A. Lanzara, P. V. Bogdanov, X. J. Zhou, S. A. Keller, D. L. Feng, E. D. Lu, T. Yoshida, H. Eisaki, A. Fujimori, K. Kishio, et al., Nature 412, 510 (2001).

[4] S. A. Trugman, J. Bonča, and L.-C. Ku, Int. J. Mod. Phys. B 15, 2707 (2001).

[5] S. Ciuchi, F. de Pascale, S. Fratini, and D. Feinberg, Phys. Rev. B 56, 4494 (1997).

[6] W. Metzner and D. Vollhardt, Phys. Rev. Lett. 62, 324 (1989).

[7] A. Georges, G. Kotliar, W. Krauth, and M. J. Rozenberg, Rev. Mod. Phys. 68, 13 (1996).

[8] A. J. Millis, R. Mueller, and B. I. Shraiman, Phys. Rev. B 54, 5389 (1996).

[9] J. Freericks and M. Jarrell, Phys. Rev. B 50, 6939 (1994).

[10] J. P. Hague and N. d'Ambrumenil, cond-mat/0106355.

[11] A. S. Alexandrov, V. V. Kabanov, and D. K. Ray, Phys. Rev. B 49, 9915 (1994).

[12] P. Benedetti and R. Zeyher, Phys. Rev. B 58, 14320 (1998).

[13] S. Blawid and A. J. Millis, Phys. Rev. B 63, 115114 (2001).

[14] A. Deppeler and A. J. Millis, Phys. Rev. B 65, 224301 (2002).

[15] H. R. Krishna-murthy, J. W. Wilkins, and K. G. Wilson, Phys. Rev. B 21, 1003 (1980).

[16] T. A. Costi, A. C. Hewson, and V. Zlatic, J. Phys.: Condens. Matter 6, 2519 (1994).

[17] O. Sakai, Y. Shimizu, and T. Kasuya, J. Phys. Soc. Japan 58, 3666 (1989).

[18] R. Bulla, Phys. Rev. Lett. 83, 136 (1999).

[19] R. Bulla, T. Costi, and D. Vollhardt, Phys. Rev. B 64, 045103 (2001).

[20] A. C. Hewson and D. Meyer, J. Phys.: Condens. Matter 14, 427 (2002).

[21] The main difficulty in extending the NRG to the model (2) lies in the unlimited number of bosonic degrees of freedom already for an isolated impurity. To perform the NRG, one has to limit the number of bosonic states. As discussed in Ref. 20], it should usually be sufficient to limit the number of allowed phonons to $\nu=4 \bar{n}=4 \frac{g^{2}}{\omega_{0}^{2}}$, where $\bar{n}$ is the average number of excited phonons. However, we usually allow $\nu=200(\gg \bar{n}$ for all situations in this paper).

[22] Note that only for $d=\infty$ this corresponds to a metalinsulator transition since here pair hopping is suppressed. 